\title{
The role of sarcomere gene mutations in patients with idiopathic dilated cardiomyopathy
}

\author{
Daniel Vega Møller ${ }^{1}$, Paal Skytt Andersen ${ }^{2}$, Paula Hedley ${ }^{2,3}$, Mads Kristian Ersbøll ${ }^{1}$, \\ Henning Bundgaard ${ }^{1}$, Johanna Moolman-Smook ${ }^{3}$, Michael Christiansen ${ }^{2}$ and Lars Køber $^{1}$
}

${ }^{1}$ Department of Cardiology, Rigshospitalet, University of Copenhagen, Denmark; ${ }^{2}$ Department of Clinical Biochemistry and Immunology, Statens Serum Institut, Copenhagen, Denmark; ${ }^{3}$ Department of Biomedical Sciences, MRC/US Centre for Molecular and Cellular Biology, University of Stellenbosch Health Sciences Faculty, Tygerberg, South Africa

We investigated a Danish cohort of 31 unrelated patients with idiopathic dilated cardiomyopathy (IDC), to assess the role that mutations in sarcomere protein genes play in IDC. Patients were genetically screened by capillary electrophoresis single strand conformation polymorphism and subsequently by bidirectional DNA sequencing of conformers in the coding regions of MYH7, MYBPC3, TPM1, ACTC, MYL2, MYL3, TNNT2, CSRP3 and TNNI3. Eight probands carried disease-associated genetic variants (26\%). In $M Y H 7$, three novel mutations were found; in MYBPC3, one novel variant and two known mutations were found; and in TNNT2, a known mutation was found. One proband was double heterozygous. We find evidence of phenotypic plasticity: three mutations described earlier as HCM causing were found in four cases of IDC, with no history of a hypertrophic phase. Furthermore, one pedigree presented with several cases of classic DCM as well as one case with left ventricular non-compaction. Disease-causing sarcomere gene mutations were found in about one-quarter of IDC patients, and seem to play an important role in the causation of the disease. The genetics is as complex as seen in HCM. Thus, our data suggest that a genetic work-up should include screening of the most prominent sarcomere genes even in the absence of a family history of the disease.

European Journal of Human Genetics (2009) 17, 1241-1249; doi:10.1038/ejhg.2009.34; published online 18 March 2009

Keywords: cardiomyopathies; dilated; heart failure; sarcomere gene mutations; DNA mutational analysis

\section{Introduction}

Dilated cardiomyopathy (DCM) is characterized primarily by left ventricular dilatation and impaired systolic function and is one of the leading causes of heart failure with high morbidity and mortality. In the absence of significant coronary artery disease or other discernible causes, its origin is considered unknown and termed idiopathic DCM (IDC). The prevalence of IDC in the United States has

Correspondence: Dr DV Møller, Department of Cardiology, Rigshospitalet, Blegdamsvej 9, 2100 Copenhagen O, Denmark.

Tel: + 45 35458619; Fax: + 45 35452549;

E-mail: dvega@gmx.net

Received 10 October 2008; revised 29 January 2009; accepted 5 February 2009; published online 18 March 2009 formerly been estimated at $36.5 / 100000$, with an incidence of $4-8 / 100000$ person-year. ${ }^{1}$ About $20-35 \%$ of the IDC cases are familial forms (FDC). ${ }^{2,3}$ FDC is defined as IDC in two or more closely related family members or, when involving a first-degree relative of an IDC patient, with a well-documented unexplained sudden death at $<35$ years of age. ${ }^{4}$ Disease-causing genes in FDC predominantly follow autosomal dominant inheritance patterns, but there have also been reports of X-linked, autosomal recessive and mitochondrial inheritance. The penetrance and presentation of FDC is highly variable concerning the functional impairment, extent of cardiac involvement and the presence of other clinical manifestations, such as woolly hair or skeletal muscle disorders. 
Genes underlying IDC have been identified from linkage as well as candidate gene studies, and include those coding for proteins involved in the cytoskeleton (desmin, ${ }^{5}$ $\delta$-sarcoglycan, ${ }^{6}$ dystrophin, ${ }^{7}$ desmoplakin $^{8}$ and metavincu$\operatorname{lin}^{9}$ ), the Z-disk (muscle LIM protein, ${ }^{10} \alpha$-actinin- $2^{11}$ and Cypher/ZASP ${ }^{12}$ ), the nuclear envelope (lamin $\mathrm{A} / \mathrm{C}^{13}$ ) and

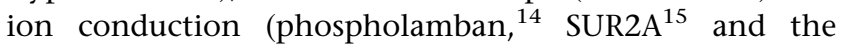
$\mathrm{Na}_{\mathrm{v}} 1.5$ ion channel ${ }^{16}$ ). Although genes encoding sarcomeric proteins were traditionally considered to be involved in hypertrophic cardiomyopathy, ${ }^{17}$ since 1998 it has also been known that mutations in these genes can cause IDC. ${ }^{18}$ Thus, mutations in the genes coding for the $\alpha$ - and $\beta$-myosin heavy chains, ${ }^{19} \alpha$-cardiac actin, ${ }^{18} \alpha$-tropomyosin, ${ }^{20}$ troponin ${ }^{2},{ }^{21}$ troponin $\mathrm{I}^{22}$ troponin $\mathrm{C},{ }^{23}$ titin, $^{24}$ myosin-binding protein $\mathrm{C}^{25}$ and telethonin ${ }^{10}$ have been shown to cause IDC.

However, the extent of sarcomeric gene involvement and its role in DCM remain largely unknown as most genetic studies in DCM have focussed on mutation screening of single genes, rather than on comprehensive screening of several sarcomeric genes. For this reason, we examined a cohort of IDC patients for mutations in nine prominent sarcomeric genes.

\section{Materials and methods}

Subjects and clinical investigations

Patients were recruited at a tertiary heart centre at the University Hospital, Rigshospitalet in Copenhagen, Denmark, and all gave written informed consent. All 31 cases were unrelated and Caucasian, with 22 FDC and 9 non-FDC index patients; all suffered from heart failure and underwent a comprehensive clinical assessment, comprising detailed clinical and familial history, physical examination, two-dimensional echocardiography, 12-lead ECG, 24-h Holter monitoring, exercise testing and standard laboratory investigations, including creatine kinase and iron studies. Coronary angiography was performed in all index patients. Patients with coronary disease, systemic arterial hypertension, primary valvular disease, pericardial disease, cor pulmonale or a history of excess alcohol consumption were excluded. The diagnostic criteria were left ventricular end-diastolic diameter (LVEDd) corresponding to a value above $3.1 \mathrm{~cm} / \mathrm{m}^{2}$ (indexed to body surface area using the Mosteller formula ${ }^{26}$ ) and fractional shortening $<25 \%$ and/or left ventricular ejection fraction (LVEF) $<45 \%$. Furthermore, we examined the hospital records of the index patients carefully to ensure that the probands represented true IDC cases and not cases of 'burnt-out' HCM. This included any history of chest pain, left ventricular hypertrophy on ECG and wall thickness in the first echocardiography performed.

Relatives of the index cases were offered a physical examination, two-dimensional echocardiography, 12-lead ECG, exercise testing and standard laboratory investigations.
Controls were 100 randomly selected, ethnically matched DNA samples extracted from Danish Guthrie cards.

The study conforms to the principles outlined in the Declaration of Helsinki and was approved by the Scientific Ethics Committee of Copenhagen and Frederiksberg (No.(KF) 01263289).

\section{Molecular genetic studies}

Patients were genetically screened at Statens Serum Institut, Copenhagen, for variants in the coding regions of the genes MYH7 (GenBank accession no. M57965.1), MYBPC3 (GenBank accession no. U91629.1), MYL2 (GenBank accession no. NG_007554.1), MYL3 (GenBank accession no. NG_007555.1), TPM1 (GenBank accession no. NG_007557.1), TNNT2 (GenBank accession no. NM_001001430.1), ACTC (GenBank accession no. NG_007553.1), CSRP3 (GenBank accession no. NM_003476) and TNNI3 (GenBank accession no. X90780.1). All patients were screened in LMNA (GenBank accession no. NM_170707.1) without discovery of any variants in the probands with sarcomere gene mutations.

Genomic DNA was isolated from whole blood samples (Qiagen, Germany). Intron-specific primers (available upon request) were used to amplify the coding regions by PCR. Amplified products were screened for mutations by capillary electrophoresis single strand conformation polymorphism $^{27}$ and by subsequent bidirectional DNA sequencing of aberrant conformers on an ABI 3100 (Applied Biosystems, Foster City, CA, USA). The frequency of all identified variants was determined in 100 Danish controls as well as in family members of the probands.

A sequence variant was considered disease causing if (1) it resulted in a missense mutation, reading frame shift, or affected a canonical splice site; (2) it affected a conserved amino acid; (3) the mutation co-segregated with the disease in affected family members; (4) it was not identified among 100 ethnically matched controls, and (5) it was not described earlier as a polymorphism in dbSNP (www.ncbi.nlm.nih.gov/SNP) or the Harvard Sarcomere mutation database (www.cardiogenomics.org) or (6) if the mutation had been described earlier as disease causing. In the absence of more than two affected family members for segregation analysis, disease association was presumed if all other criteria were met.

The effect of intronic variants on the splicing of adjacent exons was analysed in silico by NetGene2 (www.cbs.dtu.dk/services/NetGene2/) using default parameters.

\section{Results}

\section{Cohort characteristics}

The group consisted of 22 men and 9 women, with a mean LVEF of $19.2 \pm 7.8$ and LVEDd of $71.5 \pm 9.5 \mathrm{~mm}$, with a 
mean age at the time of diagnosis of $41.5 \pm 8.5$ years. Twentytwo $(71 \%)$ of them underwent HTX before or soon after inclusion in the study. In 61\% (19 probands), myocardial biopsy was performed, stained with haematoxylineosin and Masson's trichrome, revealing severe hypertrophy of the myocyte fibers as well as fibrosis, no disarray or other signs of HCM, and consistence with DCM. Maximal wall thickness of the left ventricular septal and posterior wall in all index patients did not exceed $11 \mathrm{~mm}$, evaluated by echocardiography performed at the onset of symptoms before inclusion or at the time of inclusion.

\section{Mutation screening}

In eight probands (26\%), we identified at least one heterozygous disease-causing sarcomeric gene mutation; these included 5 of 22 FDC cases (23\%) and 3 of 9 non-FDC cases (33\%). No homozygous mutation carriers were found. Individual mutations and their segregation within families are described below and in Table 1 . In one case, the index case was a double heterozygote, carrying a mutation described earlier in MYBPC3 and a novel rod mutation in MYH7. Another index case was carrying a known TNNT2 disease-causing mutation as well as two novel intronic variants of unknown significance in MYH7 and MYBPC3. No mutations were found in the other genes examined. None of the variants described below were present in 100 Danish control samples. All missense mutations involved amino acids that are evolutionarily highly conserved, as shown in Figure 1.

\section{Phenotypic description and genotype analysis}

Clinical data of probands and their mutation-positive family members are shown in Table 2, whereas IDC pedigrees and sequence data are shown in Figure 2.

Family M1 The severity of cardiac involvement within this FDC family varied from mild symptoms and discrete left ventricular dilatation to severe heart failure requiring HTX (see Table 2). The age of onset of symptoms ranged from 12 to 41 years. There was a family history of sudden cardiac death. Two family members required HTX, at 20 and 23 years of age, respectively, whereas three are stable on medical treatment. One of the members (M1, II:2) was diagnosed as having left ventricular non-compaction (LVNC) based on echocardiography. The proband's father (M1, II:3) died suddenly at 36 years of age, 2 years after being diagnosed with DCM. The proband with LVNC was diagnosed when clinically unaffected, due to a health check-up at work, by noting a left bundle branch block on her ECG. Echocardiography revealed LVNC and she has had a slow progression towards heart failure and an increasing dilatation of her left ventricle.

All affected members carried a novel MYH7 K637E mutation, as did one 34-year-old individual (M1, III:2), with normal echocardiography. This mutation results in the exchange of a highly conserved basic amino acid with an acidic amino acid. As residue 637 is located in the myosin loop, which interacts with actin (residues 626-646), the K637E mutation may affect cross-bridge formation.

Family M9 The index case of this non-FDC family had onset of symptoms a few days after giving birth to her first child. She had a short, aggressive disease period, which resulted in HTX within 3 months of onset. Both parents were older than 80 years, had no cardiovascular symptoms and declined participation in the study.

This index case carried a novel MYBPC3 T494I mutation, which replaces a conserved hydrophilic side chain with a branched neutral side chain in domain C3 of cardiac myosin-binding protein $\mathrm{C}$ (cMyBPC); such an alteration may interfere with the secondary structure of the C3 domain. The missense mutations G490R, R495Q and $\mathrm{R} 502 \mathrm{~W}$, all located in the proximity of the 494 residue, have all been described as associated with HCM.

Family M20 The female index case of this family progressed slowly from onset of symptoms at the age of 42 years, and received HTX in her sixth decade. Shortly after giving birth to her, her mother died of a suspected but unconfirmed cardiac cause; thus, this was regarded as a non-FDC case. Two healthy sisters declined participation in the study.

This index case carried the novel MYH7 K637E mutation, as described in the M1 family.

Family M21 The proband of this family (M21, II:2) had onset of symptoms at 42 years, LBBB on ECG and required cardiac resynchronization therapy. His father had onset of symptoms at 77 years of age and is stable on medication. Two asymptomatic young children (M21, III:1 and :2) had no signs of hypertrophy or dilatation on echocardiography and normal ECG.

Both affected individuals, as well as the children of the proband, carried the MYBPC3 R326Q mutation, which has been described elsewhere both as an HCM-causing mutation $^{17,28}$ and as a polymorphism associated with HCM. ${ }^{29,30}$ It results in the replacement of a conserved charged arginine with neutral glutamine at residue 326 in the phosphorylatable MyBPC motif of cMyBPC. This charge change is likely to cause functional impairment.

Family M25 In this non-FDC family, the male index case had late-onset IDC, with a slow progression to severe heart failure and subsequently HTX. He also suffered from atrial fibrillation in the later stages of his disease. None of his five siblings were affected, judged by their clinical presentation, nor was there any history of cardiac disease or sudden death in the family.

The index patient carried a known TNNT2 K247R mutation as well as two novel intronic variants in $\mathrm{MYH7}$ 
Table 1 Mutations and variants identified in IDC probands - mutations and genetic variants found in IDC patients, but not in controls, in this study

\begin{tabular}{|c|c|c|c|c|c|c|}
\hline & Mutation & Conservation & $A A$ effect & $\begin{array}{l}\text { Molecular/biophysical } \\
\text { effect }\end{array}$ & Family & Reference/disease \\
\hline \multirow[t]{4}{*}{ MYBPC3 } & & & & & & \\
\hline & g.7360G $>A$ & Complete & p.R326Q & $\begin{array}{l}\text { Loss of +charge in } \\
\text { domain C2 }\end{array}$ & M21, M57 & $\begin{array}{l}\text { Richard et } a l^{17} \text { and } \\
\text { Morner et } a^{28} / \mathrm{HCM} \text {; } \\
\text { Niimura et }\left.a\right|^{99} \text { and } \\
\text { Jaakelainen et a } a^{30} / \\
\text { HCM polymorphism; } \\
\text { this study/IDC }\end{array}$ \\
\hline & g.10925C > T & Complete & p.T494I & $\begin{array}{l}\text { Removal of }-\mathrm{OH} \text { group } \\
\text { and } \\
\text { introduction of } \\
\text { branched AA } \\
\text { in domain } \mathrm{C} 3\end{array}$ & M9 & This study/IDC \\
\hline & g.12470G >A & High & p.E619K & $\begin{array}{l}\text { Charge change in } \\
\text { domain } C 4\end{array}$ & M29 & $\begin{array}{l}\text { www.cardiogenomics. } \\
\text { org/HCM; this } \\
\text { study/IDC }\end{array}$ \\
\hline \multirow[t]{4}{*}{ MYH7 } & & & & & & \\
\hline & g. $12162 \mathrm{~A}>\mathrm{G}$ & High & p.K637E & $\begin{array}{l}\text { Charge change in } \\
\text { actin cleft }\end{array}$ & M1, M20 & This study/IDC \\
\hline & g. $17114 \mathrm{~T}>\mathrm{C}$ & High & p.L1038P & $\begin{array}{l}\text { Introduction of helix- } \\
\text { breaking } \\
\text { proline in rod }\end{array}$ & M57 & This study/IDC \\
\hline & g. $24367 C>T$ & Complete & p.R1832C & $\begin{array}{l}\text { Loss of + charge in } \\
\text { LMM and } \\
\text { introduction of } \\
\text { reactive cysteine }\end{array}$ & M52 & This study/IDC \\
\hline TNNT2 & g. $12750 \mathrm{~A}>\mathrm{G}$ & Complete & p.K247R & $\begin{array}{l}\text { Introduction of the } \\
\text { less-reactive arginine, } \\
\text { in the } \alpha \text {-helical } \\
\text { C-terminal region of } \\
\text { troponin T, } \\
\text { which interacts with } \\
\text { troponin I }\end{array}$ & M25 & $\begin{array}{l}\text { Garcia-Castro et } a^{\beta^{32} /} \\
\text { HCM; this } \\
\text { study/IDC }\end{array}$ \\
\hline \multicolumn{7}{|c|}{ Genetic variants of uncertain significance } \\
\hline MYBPC3 & \multicolumn{2}{|c|}{ g. $15972 \mathrm{~T}>\mathrm{A}$} & IVS25+13T $>A$ & Affects splicing? & M25 & This study/IDC \\
\hline MYH7 & \multicolumn{2}{|c|}{ g. $25677 C>A$} & IVS39+7C >A & Affects splicing? & M25 & This study/IDC \\
\hline
\end{tabular}

AA, amino acid; $g$, genomic; $p$, protein; LMM, light meromyosin.

a Potential altered splicing predicted by NetGene2.

(IVS39 + 7C > A) and MYBPC3 (IVS25 + 13T > A). Neither of the two novel intronic variants was found in the controls nor in dbSNP or Ensembl.

The MYBPC3 variant was not found to interfere with splicing in silico, whereas MYH7 (IVS39 + 7C > A) increased the likelihood of a cryptic donor splice site to $70 \%$, possibly resulting in the synthesis of mRNA coding for a $\beta$-myosin with a $C$ terminus of -VGLE instead of -GLNEE. As the variant is located in the last exon, the faulty spliced mRNA is probably not degraded but results in the synthesis of a modified protein. ${ }^{31}$ The variant could thus possibly result in the synthesis of a limited amount of the slightly modified $\beta$-myosin. The functional significance of this is not easily established, but it suggests that the variant may be a disease modifier.

The TNNT2 K247R mutation has formerly been described as an HCM-causing mutation. ${ }^{32}$ The mutation substitutes a lysine with a more bulky arginine in the $\alpha$-helical C-terminal region of troponin $\mathrm{T}$, which interacts with troponin I. This residue is close to another HCM-associated TNNT2 mutation, E244D, which replaces a bulky glutamate residue with a less bulky aspartate residue. In vitro experiments have suggested that the E244D mutation interferes with both $\mathrm{Ca}^{2+}$ sensitization and the maximum 
MYBPC3

Homo_sapiens

Pan troglodytes

Canis_familiars

Bos_täurus

Mus musculus

RatẼus norvegicus

Monodelphis_domestica

Tupaia belangeri

\section{MYH7}

Homo sapiens

Felis_catus

Mus músculus

Rattus norvegicus

Monodelphis_domestica

TNNT2

Homo_sapiens

Pan Eroglodytes

Erinaceus europaeus

Rattus_norvegicus

Mus muśculus

Gallus gallus

Felis_catus

Tupaiābelangeri
R326Q

VWEILRQAPPS... VWEILRQAPPS...

VWEILRQASPS...

VWEILRQAPPS..

VWEILRQAPPS...

VWEILRQAPPS...

VWEILRTAPPS...

VWALLGQAPPS...

$\star \star: * * . * * \ldots$

K637E

EKGKGKAKKGS...

DKGKGKAKKGS...

DKGKGKAKKGS...

DKGKGKAKKGS..

AKGKGGKKKGS...

$\star * \star *, * * * *$

K247R

FDLQEKFKQQK

FDLQEKFKQQK

FDLQEKFKQQK

FDLQEKFKQQK

FDLQEKFKQQK

FDLQEKFKRQK

FDLQEKFKQQK

FDLQEKFKQQK

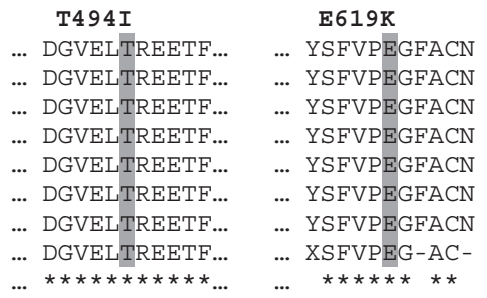

\begin{tabular}{|c|c|}
\hline L10 & R1832C \\
\hline ...DLEGSLEQEKK.. & ...EAEQKRNAESV \\
\hline ...DLEGSLEQEKK.. & ...EAEQKRNAESV \\
\hline ...DLEGSLEQEKK.. & ...EAEQKRNAESV \\
\hline ...DLEGSLEQEKK.. & ...EAEQKRNAESV \\
\hline ...DLEGSLEQEKK.. & ...ESEQKRNVESV \\
\hline & \\
\hline
\end{tabular}

Figure 1 Homology analysis of missense mutations - cross-species comparison of protein regions in which missense mutations occur. All mutations affected highly conserved residues.

level of myosin ATPase activity. ${ }^{33}$ Thus, it is plausible that the K247 mutation also affects the troponin T/troponin I interaction and, in consequence, the $\mathrm{Ca}^{2+}$-mediated control of contractility.

Family M29 This FDC family had two affected heterozygous brothers with onset of symptoms in their third decade; the index case required HTX 3 years after the onset of symptoms. Three unaffected children were examined (IV:1, IV:2 and IV:3), all with normal echocardiography without signs of hypertrophy or dilatation.

Both affected individuals as well as a 26-year-old unaffected daughter (M29, IV:3) carried the MYBPC3 E619K mutation. This mutation has been described earlier (www.cardiogenomics.org) as associated with HCM, but no clinical data were reported. A nearby mutation, A627V, has also been associated with HCM, but only in a homozygous carrier, whereas two A627V heterozygotes were asymptomatic. ${ }^{34}$ The E619K mutation results in a charge change in the C4 domain of cMyBPC and may affect the secondary structure of the domain.

Family M52 The proband in this FDC family had an early onset of symptoms, in his third decade, with severe dilatation of his left ventricle and depressed LVEF, and required HTX 3 years after the onset of symptoms. His father died at the age of 47 years due to IDC, whereas a sibling of the index case died suddenly within her first year. It was not possible to obtain DNA from the deceased relatives.

We found a novel MYH7 R1832C in the index case. This mutation is located in the rod portion of MYH7 and replaces a basic arginine residue with a reactive neutral cysteine residue in the g-position of the heptad repeat in the $\alpha$-helical myosin rod, and thus may destabilize the salt bridges required for myosin-myosin dimerization.

Family M57 The index case in this family received HTX at 50 years of age (14 years after the onset of symptoms) after a long stable period on medication. He also developed atrial fibrillation, well regulated on medication. His father died due to IDC in his fourth decade, whereas his brother developed IDC. DNA was not available from either of these individuals.

The index case was double heterozygous for the known MYBPC3 R326Q mutation, also described in the M21 family, and had a novel rod mutation, L1038P, in MYH7. The L1038P mutation results in the substitution of leucine with a helix-breaking proline in the core ' $a$ ' position of the heptad repeat motif in the $\alpha$-helical S2 region of the myosin molecule, which is likely to affect its secondary structure and coiled-coil formation. 


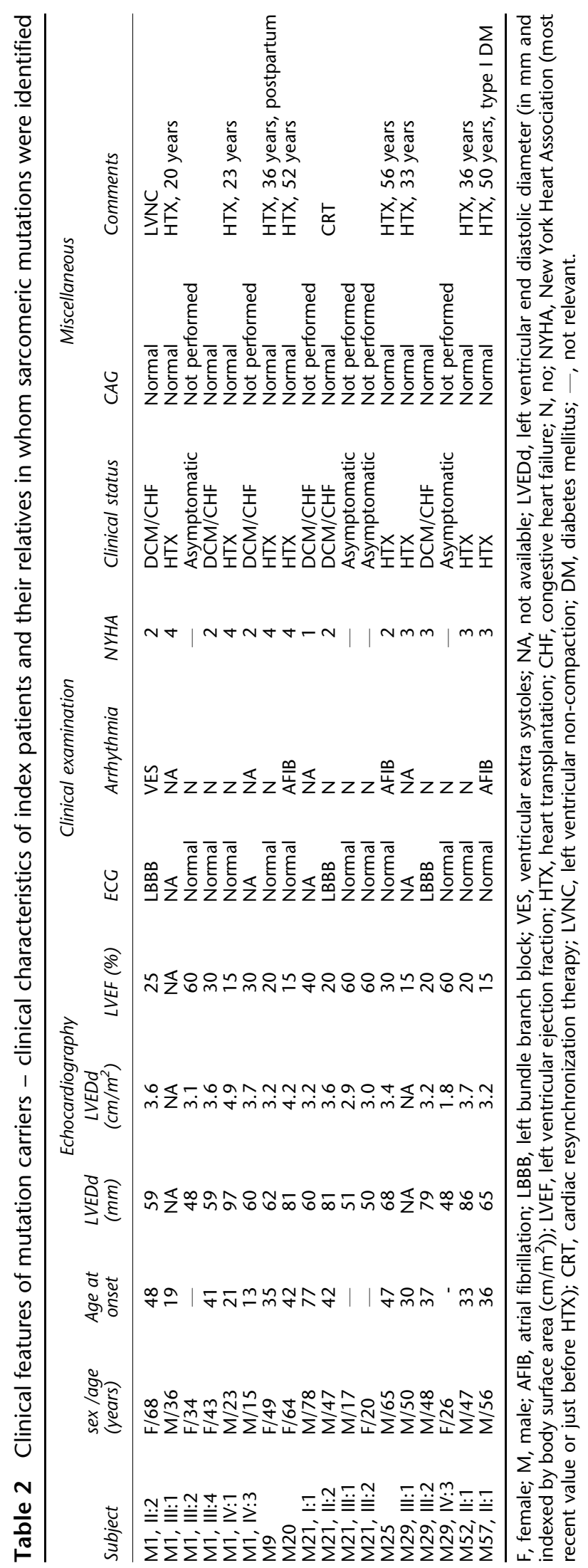

\section{Discussion}

Among the 31 IDC cases investigated in this study, we found $26 \%$ (8) to be caused by mutations in three sarcomeric genes; these included four new disease-causing mutations, as well as three that have been described earlier as causing HCM, namely, the K247R in TNNT2, and the E619K and R326Q in MYBPC3. Although the latter variant has also been described as a polymorphism in two earlier studies, ${ }^{29,30}$ the other two mutations have been described only as HCM-causing mutations, indicating phenotypic plasticity.

The mutations described in this study were found in five patients with and three patients without a family history of IDC. We found a frequency of sarcomeric gene mutations in IDC that is much higher than that the earlier studies have suggested, ${ }^{21}$ and this is only slightly less than that found in HCM overall (familial and sporadic cases), ${ }^{28,35}$ making mutation screening of prominent sarcomere genes clinically relevant in IDC. Our finding that 2 out of $8(25 \%)$ probands carried more than one suspected disease-causing mutation suggests that the frequency of compound sarcomeric gene mutations is also similar between the two cardiomyopathies, and indicates the need for a comprehensive gene screening in DCM, as in HCM.

Although the same sarcomeric genes have been implicated in both HCM and IDC as well as LVNC, to our knowledge, the same plasticity has not yet been found concerning the same sarcomeric mutation, although phenotypic plasticity involving HCM and IDC has been described for a metavinculin missense mutation. ${ }^{36}$ From hospital follow-up records, early echocardiographic recordings and gross and histological examination of explanted hearts, we found no evidence to suggest that any of the index cases carrying either the K247R in TNNT2 or the E619K and R326Q in MYBPC3 (families M21, M25, M29 and M57) in fact presented with burnt-out HCM.

Although it is accepted that different mutations in the same sarcomeric gene can cause either HCM or IDC, the mechanism precipitating either cardiomyopathy is still unknown. The early suggestion that the location of a given mutation in particular sarcomeric protein domains may be the deciding factor giving rise to either hypertrophic or DCM has not been borne out by further studies. ${ }^{37}$ Later studies have proposed that the difference between HCMand IDC-causing mutations lies in the functional effects the distinct mutations in the same gene have on contractility. ${ }^{38,39}$ However, this functional-effect hypothesis would not account for phenotypical plasticity of the same mutations, such as those described here. One possible explanation for this phenotypic plasticity is that the effect of some mutations is modified by other unknown genetic or environmental factors. Alternatively, these mutations, which are apparently pathological in their own right, may, in fact, themselves be modifiers of other unknown, 'more pathological' mutations, and the conjunction of these 

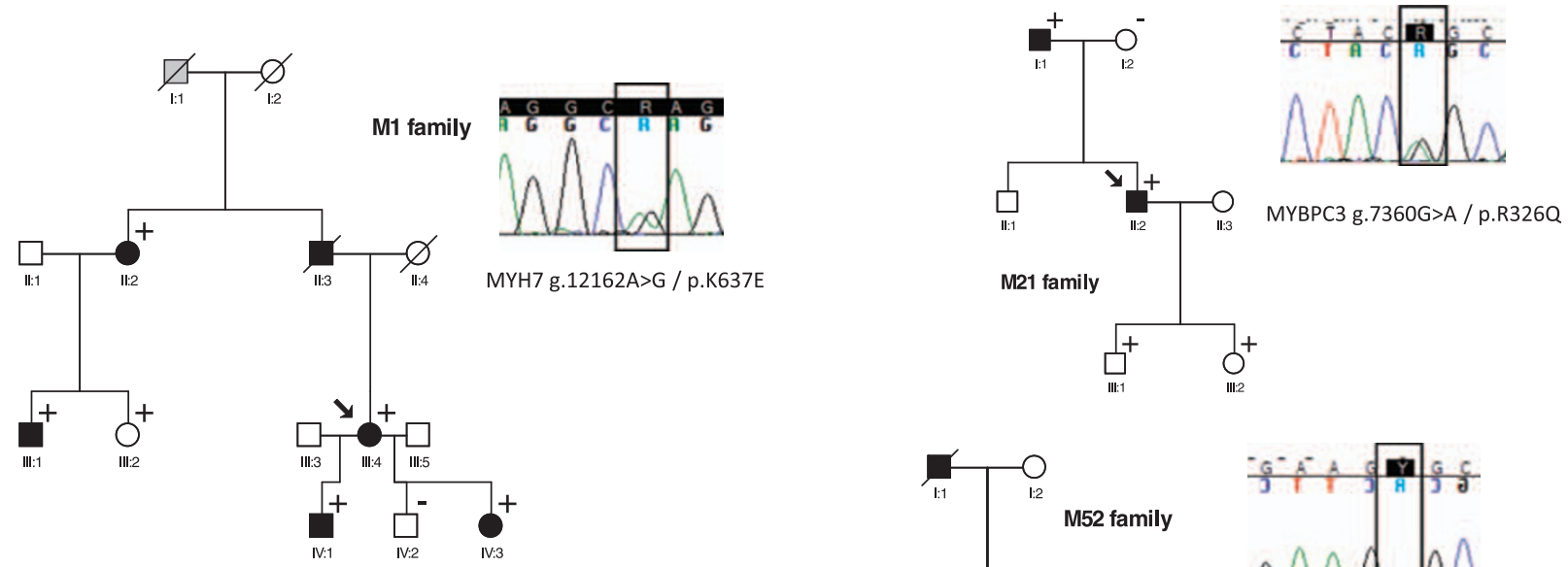

M21 family
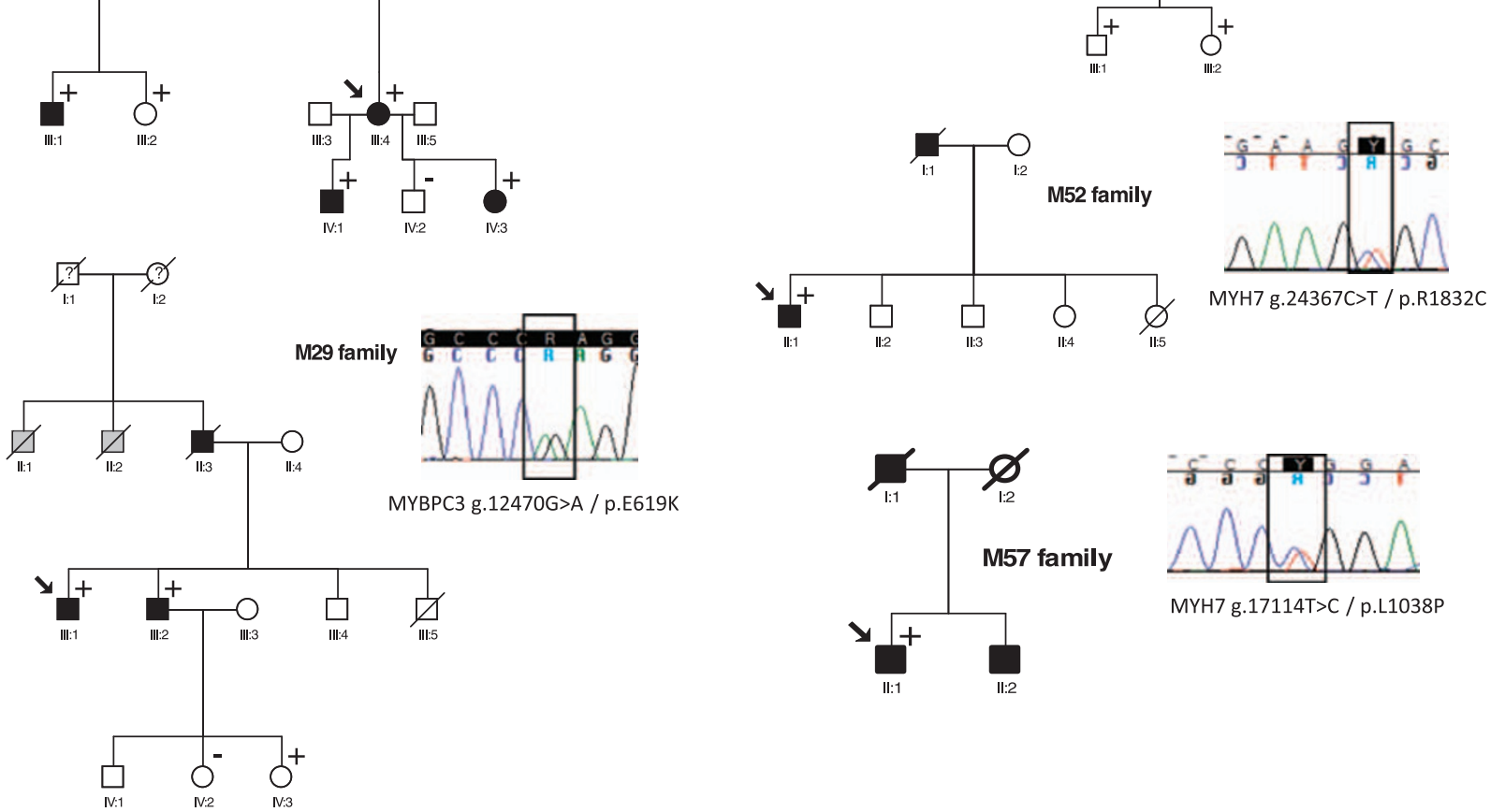

MYBPC3 g.12470G>A / p.E619K
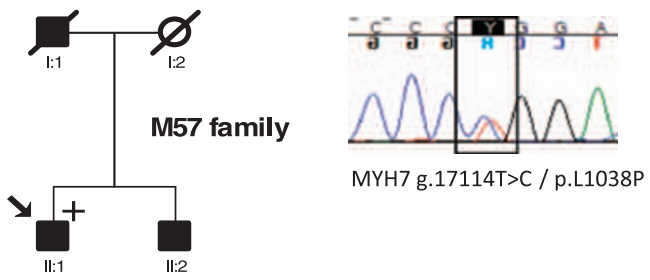

Figure 2 Pedigrees of FDC families with mutations and sequencing data - drawn are FDC pedigrees and their corresponding sequence mutation. Arrow indicates the proband. Solid symbols indicate documented affected individuals, grey symbols indicate suspected dilated cardiomyopathy and open symbols indicate unaffected individuals. Mutation carrier is indicated by a + sign, and subjects without mutation are indicated by a - sign Slashed symbols indicate deceased members. Solid box in the sequence data indicates mutation site at the sequence.

various effects, and the size of these effects including environmental modifying effects, is what precipitates the particular cardiomyopathy in any given person. This could also explain the asymptomatic mutation carriers seen within the pedigrees, although the onset of symptoms in IDC often appears in the third to fourth decade of life, which only a few of them have reached.

The MYBPC3 R326Q mutation seen in two unrelated families (M21 and M57) appears to be such a modifier. Jaaskelainen et $\mathrm{l}^{30}$ found this variant in one HCM patient, but also in 7 out of 111 controls, whereas both Morner et $a l^{28}$ and Richard et $a l^{17}$ each found it in one HCM patient in their respective cohorts, but not in 400 controls. In our study, it occurred in two FDC families (M21 and M57), but not in 100 controls. In family M21, the mutation segregated with the disease, whereas in M57, the R326Q variant co-occurred with a novel MYH7 rod mutation, L1038, in the index case, who showed a slow progression of
IDC. A cross-species comparison of cMyBPC amino-acid sequences shows a high degree of conservation at this position (Figure 1), and the change in charge brought about by the R326Q suggests that it may have functional consequences, but it could be speculated that these consequences are not sufficient to cause pathology in the absence of a second genetic or environmental hit. So it could be suggested that the R326Q variant should not be included with the rest of the mutations, although it conforms to our mutation criteria set in the Materials and methods section. If this was the case only $23 \%$ of the index patients suffered from sarcomeric gene mutations.

Further support for the interaction between an apparently pathological sarcomeric mutation and additional genetic and environmental modifiers and the precipitation of IDC lies in the wide spectrum of cardiac involvement present in family M1. In this family, the onset of symptoms ranges from the second to the fifth decade, and involves 
LVNC in one individual and classical DCM phenotypes in others. A recent study also found sarcomere gene mutations to be responsible for $\mathrm{LVNC}^{40}$ and thus altogether it has widened the spectrum of different cardiomyopathies triggered by mutations in the same sarcomere genes.

The consequences of genetic testing involve the affected individual as well as the relatives, and the benefits of genetic testing are (1) confirmation of diagnosis in patients, (2) early detection and potential prevention in relatives and (3) the exclusion of causative mutation in relatives. The result can either terminate clinical follow-up of relatives or institute follow-up.

Identification of disease-causing mutations in relatives may influence the ability/advisability to perform physical activity at competition level; to acquire life insurance; and to pursue the preferred professional career although guidelines concerning these issues are at present not available and may turn out to be gene specific. Furthermore, the institution of anti-congestive medication involved in remodelling (ie, ACE inhibitors and $\beta$-blockers) to asymptomatic mutation carriers with only a slight left ventricular function impairment is believed to preserve left ventricular function and extend the asymptomatic state as seen in large randomized trials. ${ }^{41,42}$ However, concerning both strategies involving modification of lifestyle and treatment options, the clinical utility must be assessed in prospective clinical trials. Furthermore, the clinical utility of screening DCM patients for sarcomere gene mutations may be less obvious in other populations with a lower frequency of disease-associated genetic variants.

The index patients were all recruited at a tertiary centre and could represent more severe cases of heart failure. As a consequence, it could be speculated that it might lead to a higher percentage of probands with causative mutations. The sample size was limited to 31 index patients due to the comprehensive nature of the genetic screening performed. Despite the limited statistical power of the study due to the sample size, this study is significant as it represents the result of this comprehensive sarcomere gene screening. Functional studies could have elucidated the consequences of a given mutation and perhaps provided a better understanding of the discordance between aetiology and clinical presentation, as well as the variable expressivity and reduced penetrance seen within the pedigrees. The asymptomatic mutation carriers seen could be explained by a late penetration of the disease or, as speculated above, by some of the mutations acting as modifiers.

In conclusion, our study, considering the sample size, indicates that sarcomere gene mutations seem to play an important role in both FDC and non-FDC. Furthermore, our data suggest that mutation screening should not be limited to clear-cut FDC cases only, but due to our limited sample size, the precise clinical utility awaits future larger prospective trials. Sarcomere gene mutations are responsible for a diversity of cardiac morphologies, including
DCM and LVNC. Furthermore, known HCM-causing mutations are found in probands with no hypertrophic phase, indicating phenotypic plasticity. Finally, sarcomere screening should not be stopped unless at least MYH7, MYBPC3 and TNNT2 sarcomere genes have been screened, due to the complex genetics of IDC, although phenotypic plasticity may complicate the interpretation of the results.

\section{Acknowledgements}

The study was supported by The Danish Heart Foundation (Grant no. 05-10-B323-A735-22257), The John and Birthe Meyer Foundation, and The Eva and Henry Fraenkel Foundation. We thank Mette Hougaard and Kirsten Lindboe for their laboratory skills.

\section{Conflict of interest}

None declared.

\section{References}

1 Codd MB, Sugrue DD, Gersh BJ, Melton III LJ: Epidemiology of idiopathic dilated and hypertrophic cardiomyopathy. A population-based study in Olmsted County, Minnesota, 1975-1984. Circulation 1989; 80: 564-572.

2 Grunig E, Tasman JA, Kucherer H, Franz W, Kubler W, Katus HA: Frequency and phenotypes of familial dilated cardiomyopathy. J Am Coll Cardiol 1998; 31: 186-194.

3 Michels VV, Moll PP, Miller FA et al: The frequency of familial dilated cardiomyopathy in a series of patients with idiopathic dilated cardiomyopathy. $N$ Engl J Med 1992; 326: 77-82.

4 Mestroni L, Maisch B, McKenna WJ et al: Guidelines for the study of familial dilated cardiomyopathies. Collaborative Research Group of the European Human and Capital Mobility Project on Familial Dilated Cardiomyopathy. Eur Heart J 1999; 20: 93-102.

5 Li D, Tapscoft T, Gonzalez O et al: Desmin mutation responsible for idiopathic dilated cardiomyopathy. Circulation 1999; 100: 461-464.

6 Tsubata S, Bowles KR, Vatta M et al: Mutations in the human delta-sarcoglycan gene in familial and sporadic dilated cardiomyopathy. J Clin Invest 2000; 106: 655-662.

7 Muntoni F, Di LA, Porcu M et al: Dystrophin gene abnormalities in two patients with idiopathic dilated cardiomyopathy. Heart 1997; 78: 608-612.

8 Norgett EE, Lucke TW, Bowers B, Munro CS, Leigh IM, Kelsell DP: Early death from cardiomyopathy in a family with autosomal dominant striate palmoplantar keratoderma and woolly hair associated with a novel insertion mutation in desmoplakin. J Invest Dermatol 2006; 126: 1651-1654.

9 Olson TM, Illenberger S, Kishimoto NY, Huttelmaier S, Keating MT, Jockusch BM: Metavinculin mutations alter actin interaction in dilated cardiomyopathy. Circulation 2002; 105: $431-437$.

10 Knoll R, Hoshijima M, Hoffman HM et al: The cardiac mechanical stretch sensor machinery involves a $\mathrm{Z}$ disc complex that is defective in a subset of human dilated cardiomyopathy. Cell 2002; 111: 943-955.

11 Mohapatra B, Jimenez S, Lin JH et al: Mutations in the muscle LIM protein and alpha-actinin-2 genes in dilated cardiomyopathy and endocardial fibroelastosis. Mol Genet Metab 2003; 80: 207-215.

12 Vatta M, Mohapatra B, Jimenez S et al: Mutations in Cypher/ZASP in patients with dilated cardiomyopathy and left ventricular noncompaction. J Am Coll Cardiol 2003; 42: 2014-2027. 
13 Fatkin D, MacRae C, Sasaki T et al: Missense mutations in the rod domain of the lamin A/C gene as causes of dilated cardiomyopathy and conduction-system disease. N Engl J Med 1999; 341: $1715-1724$.

14 Schmitt JP, Kamisago M, Asahi M et al: Dilated cardiomyopathy and heart failure caused by a mutation in phospholamban. Science 2003; 299: 1410-1413.

15 Bienengraeber M, Olson TM, Selivanov VA et al: ABCC9 mutations identified in human dilated cardiomyopathy disrupt catalytic KATP channel gating. Nat Genet 2004; 36: 382-387.

16 Olson TM, Michels VV, Ballew JD et al: Sodium channel mutations and susceptibility to heart failure and atrial fibrillation. JAMA 2005; 293: 447-454.

17 Richard P, Charron P, Carrier L et al: Hypertrophic cardiomyopathy: distribution of disease genes, spectrum of mutations, and implications for a molecular diagnosis strategy. Circulation 2003; 107: 2227-2232.

18 Olson TM, Michels VV, Thibodeau SN, Tai YS, Keating MT: Actin mutations in dilated cardiomyopathy, a heritable form of heart failure. Science 1998; 280: 750-752.

19 Villard E, Duboscq-Bidot L, Charron P et al: Mutation screening in dilated cardiomyopathy: prominent role of the beta myosin heavy chain gene. Eur Heart J 2005; 26: 794-803.

20 Olson TM, Kishimoto NY, Whitby FG, Michels VV: Mutations that alter the surface charge of alpha-tropomyosin are associated with dilated cardiomyopathy. J Mol Cell Cardiol 2001; 33: $723-732$.

21 Kamisago M, Sharma SD, DePalma SR et al: Mutations in sarcomere protein genes as a cause of dilated cardiomyopathy. N Engl J Med 2000; 343: 1688-1696.

22 Murphy RT, Mogensen J, Shaw A, Kubo T, Hughes S, McKenna WJ: Novel mutation in cardiac troponin I in recessive idiopathic dilated cardiomyopathy. Lancet 2004; 363: 371-372.

23 Mogensen J, Murphy RT, Shaw T et al: Severe disease expression of cardiac troponin $\mathrm{C}$ and $\mathrm{T}$ mutations in patients with idiopathic dilated cardiomyopathy. J Am Coll Cardiol 2004; 44: 2033-2040.

24 Gerull B, Gramlich M, Atherton J et al: Mutations of TTN, encoding the giant muscle filament titin, cause familial dilated cardiomyopathy. Nat Genet 2002; 30: 201-204.

25 Daehmlow S, Erdmann J, Knueppel T et al: Novel mutations in sarcomeric protein genes in dilated cardiomyopathy. Biochem Biophys Res Commun 2002; 298: 116-120.

26 Mosteller RD: Simplified calculation of body-surface area. $N$ Engl J Med 1987; 317: 1098.

27 Andersen PS, Jespersgaard C, Vuust J, Christiansen M, Larsen LA: High-throughput single strand conformation polymorphism mutation detection by automated capillary array electrophoresis: validation of the method. Hum Mutat 2003; 21: 116-122.

28 Morner S, Richard P, Kazzam E et al: Identification of the genotypes causing hypertrophic cardiomyopathy in northern Sweden. J Mol Cell Cardiol 2003; 35: 841-849.

29 Niimura H, Patton KK, McKenna WJ et al: Sarcomere protein gene mutations in hypertrophic cardiomyopathy of the elderly. Circulation 2002; 105: 446-451.
30 Jaaskelainen P, Kuusisto J, Miettinen $\mathrm{R}$ et al: Mutations in the cardiac myosin-binding protein $\mathrm{C}$ gene are the predominant cause of familial hypertrophic cardiomyopathy in eastern Finland. J Mol Med 2002; 80: 412-422.

31 Wagner E, Lykke-Andersen J: mRNA surveillance: the perfect persist. J Cell Sci 2002; 115 (Part 15): 3033-3038.

32 Garcia-Castro M, Reguero JR, Batalla A et al: Hypertrophic cardiomyopathy: low frequency of mutations in the beta-myosin heavy chain (MYH7) and cardiac troponin T (TNNT2) genes among Spanish patients. Clin Chem 2003; 49: 1279-1285.

33 Yanaga F, Morimoto S, Ohtsuki I: $\mathrm{Ca}^{2+}$ sensitization and potentiation of the maximum level of myofibrillar ATPase activity caused by mutations of troponin $\mathrm{T}$ found in familial hypertrophic cardiomyopathy. J Biol Chem 1999; 274: $8806-8812$.

34 Garcia-Castro M, Reguero JR, Alvarez V et al: Hypertrophic cardiomyopathy linked to homozygosity for a new mutation in the myosin-binding protein $\mathrm{C}$ gene $(\mathrm{A} 627 \mathrm{~V})$ suggests a dosage effect. Int J Cardiol 2005; 102: 501-507.

35 Jaaskelainen P, Miettinen R, Karkkainen P, Toivonen L, Laakso M, Kuusisto J: Genetics of hypertrophic cardiomyopathy in eastern Finland: few founder mutations with benign or intermediary phenotypes. Ann Med 2004; 36: 23-32.

36 Vasile VC, Will ML, Ommen SR, Edwards WD, Olson TM, Ackerman MJ: Identification of a metavinculin missense mutation, R975W, associated with both hypertrophic and dilated cardiomyopathy. Mol Genet Metab 2006; 87: 169-174.

37 Nanni L, Pieroni M, Chimenti C et al: Hypertrophic cardiomyopathy: two homozygous cases with 'typical' hypertrophic cardiomyopathy and three new mutations in cases with progression to dilated cardiomyopathy. Biochem Biophys Res Commun 2003; 309: 391-398.

38 Robinson P, Griffiths PJ, Watkins H, Redwood CS: Dilated and hypertrophic cardiomyopathy mutations in troponin and alphatropomyosin have opposing effects on the calcium affinity of cardiac thin filaments. Circ Res 2007; 101: 1266-1273.

39 Debold EP, Schmitt JP, Patlak JB et al: Hypertrophic and dilated cardiomyopathy mutations differentially affect the molecular force generation of mouse alpha-cardiac myosin in the laser trap assay. Am J Physiol Heart Circ Physiol 2007; 293: $\mathrm{H} 284-\mathrm{H} 291$.

40 Klaassen S, Probst S, Oechslin E et al: Mutations in sarcomere protein genes in left ventricular noncompaction. Circulation 2008; 117: 2893-2901.

41 Jong P, Yusuf S, Rousseau MF, Ahn SA, Bangdiwala SI: Effect of enalapril on 12-year survival and life expectancy in patients with left ventricular systolic dysfunction: a follow-up study. Lancet 2003; 361: 1843-1848.

42 Remme WJ, Riegger G, Hildebrandt P et al: The benefits of early combination treatment of carvedilol and an ACE-inhibitor in mild heart failure and left ventricular systolic dysfunction. The carvedilol and ACE-inhibitor remodelling mild heart failure evaluation trial (CARMEN). Cardiovasc Drugs Ther 2004; 18: $57-66$. 